\title{
Review Article \\ Characteristic Changes of Scale Invariance of Seismicity Prior to Large Earthquakes: A Constructive Review
}

\author{
Qiang Li and Gui-Ming Xu \\ Research Center for Earthquake Prediction, Earthquake Administration of Jiangsu Province, No. 3 Wei Gang, Nanjing 210014, China \\ Correspondence should be addressed to Qiang Li; lqdzjybzx@126.com
}

Received 7 February 2013; Revised 2 May 2013; Accepted 10 June 2013

Academic Editor: Filippos Vallianatos

Copyright (C) 2013 Q. Li and G.-M. Xu. This is an open access article distributed under the Creative Commons Attribution License, which permits unrestricted use, distribution, and reproduction in any medium, provided the original work is properly cited.

\begin{abstract}
Recently, research on the characteristic changes of scale invariance of seismicity before large earthquakes has received considerable attention. However, in some circumstances, it is not easy to obtain these characteristic changes because the features of seismicity in different regions are various. In this paper, we firstly introduced some important research developments of the characteristic changes of scale invariance of seismicity before large earthquakes, which are of particular importance to the researchers in earthquake forecasting and seismic activity. We secondly discussed the strengths and weaknesses of different scale invariance methods such as the local scaling property, the multifractal spectrum, the Hurst exponent analysis, and the correlation dimension. We finally came up with a constructive suggestion for the research strategy in this topic. Our suggestion is that when people try to obtain the precursory information before large earthquakes or to study the fractal property of seismicity by means of the previous scale invariance methods, the strengths and weaknesses of these methods have to be taken into consideration for the purpose of increasing research efficiency. If they do not consider the strengths and weaknesses of these methods, the efficiency of their research might greatly decrease.
\end{abstract}

\section{Introduction}

It is a well-known fact that the natural seismic system and the rock fracture system in laboratory have the properties of scale invariance [1-13]. Study on the characteristic changes of scale invariance of seismicity before large ruptures has been an intriguing subject to geophysicists recently. So far, great progresses have been made in this topic. For instance, analysis results of the temporal and spatial multifractal characteristic of seismicity indicate that there are anomalous changes of the singularity spectrum and generalized dimension spectrum before some large earthquakes [14-20]; study results of the earthquakes [21,22], rock mechanics experiments [23, 24], and rock burst [25] indicate that the there are anomalous variations in the dimension of fractal objects prior to the major ruptures; research by $\mathrm{Li}$ and $\mathrm{Xu}$ [26] indicates that there is the possible correlation between the featuring change of the local scaling property and the process of seismogeny; a study by Zhao and Wang [27] shows that the Hurst exponent for the sequence of the interval time between earthquakes decreases prior to some large inland earthquakes. In addition, some study results proposed that the decrease of fractal dimension and Hurst exponent, as well as the characteristic change of generalized dimension spectrum, is deemed as precursory phenomena before main avalanches or ruptures [16, 28-35].

However, different scale invariance methods have their own strengths and weaknesses. Some methods can only give the description of the monofractal property of seismicity [36], while others can sufficiently give the description of heterogeneous properties of fractal seismic system [37, 38]. Besides, the research results of some scale invariance methods (such as the multifractal spectrum, the correlation dimension, the fractal dimension, and Hurst exponent analysis of seismicity) are relatively mature because there have been many studied cases which show characteristic changes of scale invariance of seismicity prior to large earthquakes, while the research results of local scaling property are only tentative [26, 3941] because the observed cases which show characteristic changes of local scaling property of seismicity prior to large earthquakes have been quite few so far.

In this paper, we introduce some important research developments of characteristic changes of scale invariance of seismicity before large earthquakes and discuss the strengths and weaknesses of different methods of scale invariance, 
for the purpose of improving the study validity for the researchers in earthquake forecasting and seismic activity.

\section{Multifractal Spectrum of Seismicity}

The multifractal spectrum includes the generalized dimension spectrum and singularity spectrum. Because the singularity spectrum is closely related to the generalized dimension spectrum, we mainly focus on generalized dimension spectrum (the curve graph of multifractal dimension $D_{q}$ versus $q$ is called the generalized dimension spectrum) in this paper.

The generalized dimension spectrum of seismicity is studied by an algorithm based on the correlative integral [38]. Computation of multifractal dimension $D_{q}$ applying the correlative integral algorithm [18] is made using

$$
D_{q}=\lim _{r \rightarrow 0} \frac{\log C_{q}(r)}{\log r}
$$

where $C_{q}(r)$ is the $q$ th-order generalized correlation function, defined as

$$
C_{q}(r)=\left\{\frac{1}{N} \sum_{j=1}^{N}\left[\frac{1}{N} \sum_{\substack{i=1 \\ i \neq j}}^{N} \Theta\left(r-\left|X_{i}-X_{j}\right|\right)\right]^{q-1}\right\}^{1 /(q-1)}
$$

with $N$ as the number of data points, $r$ as the scaling radius, $\Theta(s)$ as the Heaviside step function, and $\left|X_{i}-X_{j}\right|$ as the distance between the two data points $X_{i}$ and $X_{j}$. If the series has the property of fractals, the $C_{q}(r)$ is indicated by a power law. Thus, the value of $D_{q}$ can be settled by calculating the linear segment slope in the graph of $\log C_{q}(r)$ versus $\log r$.

Roy and Padhi [19] studied the generalized dimension spectrum of seismicity in Iran and adjacent regions. They found that the precursory clustering pattern in the short time period before three large earthquakes $\left(M_{w}=7.8\right.$ on 16.9.1978; $M_{w}=6.8$ on $26.12 .2003 ; M_{w}=7.7$ on 10.5 .97 ) is perceived by analyzing the multifractal property of seismicity in this area. They believe that the probability for future large earthquakes can be evaluated by studying the spatial and temporal clustering pattern of earthquakes. They conclude that the clustering pattern study based on seismic catalogue for majority of the known seismic fault systems may be finally helpful to the prevention and mitigation of earthquake hazards.

Caruso et al. [14] made a study on the multifractal property of Mount St. Helens seismicity during the period of 1980-2002, in which the temporal distribution of seismicity related to the eruptive activity primarily characterized by the major explosive eruptions in 1980 and the eruptions during the period of 1980-1986 is analyzed. They found that the generalized dimension spectrum, which is calculated from the data of seismicity, can help us to recognize two main temporal distribution patterns of seismicity. The first pattern shows a multifractal clustering related to the strong earthquake swarm of the dome building activity. The second pattern is typified by very small variation value of $D_{q}$ close to 1 , as for a stochastic uniform distribution. The temporal change of $D_{q}$ calculated from an invariant window length of seismic events and at different depths shows that the fragile mechanical response of the shallow zones to swift magmatic intrusions, through the whole course of the eruption, is indicated by its rapid variations in a short period and the smallest values of $D_{q}$ (about 0.3).

Dimitriu et al. [15] studied the generalized dimension spectrum in a seismically active area of Northern Greece, where several large earthquakes, containing the May 1995 Arena sequence with a maximum $M_{w} 5.3$ earthquake on May 4,1995 , occurred. They found that multifractality enhances before the major seismicity and that the clusterization is replaced by the declusterization not long before its initialization. They believe that this might help us to approximately evaluate the occurrence time of the main slip, if the multifractality getting to its peak before the percolation makes the previous gradual increase in multifractality.

Teotia and Kumar [20] studied the generalized dimension spectrum by analyzing the seismic catalog data in the North-Western Himalaya area, which primarily contain the seismogenic zone of 1905 Kangra great earthquake. The seismic catalogue data come from USGS catalogue during the period of 1973-2009, which contains the MuzaffarabadKashmir earthquake $\left(M_{w}=7.6\right)$ of October 2005. They found the temporal variations in generalized dimension spectrum before the Muzaffarabad-Kashmir earthquake in the area. They believe that this study may be of important value in recognizing the seismogenic zone of large seismic events in dissimilar tectonic regions.

Li [17] studied the variation of multifractal characteristic before and after four mid-strong earthquakes (magnitudes range from 5.5 to 6.2 ) in Jiangsu province and its adjacent area, in the east of China, by analyzing seismicity data since 1970 in this area, where the frequency and strength of seismicity belong to the middle level, and the completeness magnitude of the catalog, estimated by using GutenbergRichter formula, is 2.4. I found that the generalized dimension spectrum and singularity spectrum $f(\alpha)$ of seismicity have three variation stages which separately corresponded to the time far before mid-strong earthquakes, the time when the anomalous change of the spectrum appeared, and the time when the anomalous change of the spectrum disappeared. Figure 1 shows the three variation stages of the generalized dimension spectrum and singularity spectrum before 22 April 1974 Liyang M5.5 earthquake (the data set includes 311 earthquakes). Among the figures, Figures 1(a) and $1(d)$ show the first variation stage of the generalized dimension spectrum and singularity spectrum (two years prior to this earthquake), respectively, in which the level difference between the left end and right ends of the generalized dimension spectrum is small (smaller than 0.44). Figures 1(b) and 1(e) show the anomalous change stage (i.e., the second variation stage) of generalized dimension spectrum and singularity spectrum (seven months prior to this earthquake), respectively, in which the curve of the generalized dimension spectrum is steeper than the two others (the level difference between the left end and right ends of the generalized dimension spectrum is larger than 0.58), and the value range of the singularity spectrum is broader than the two others. Figures 1(c) and 1(f) show the third 


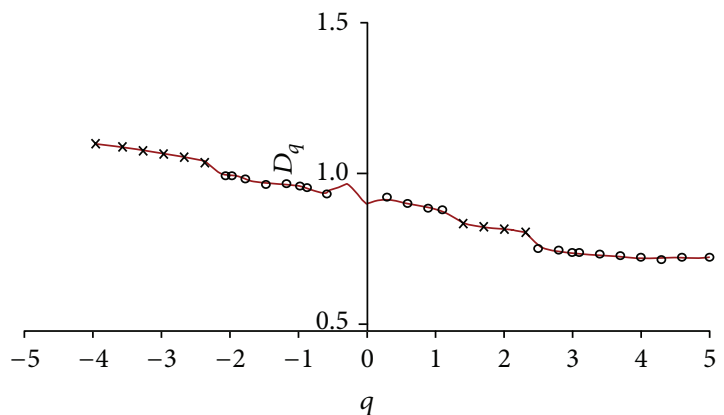

(a)

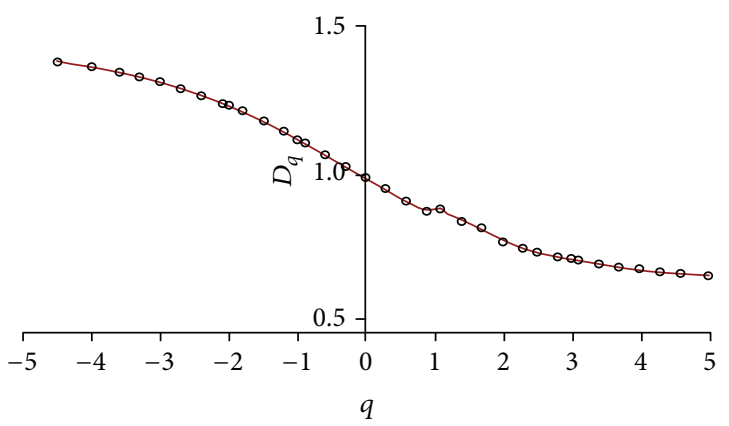

(b)

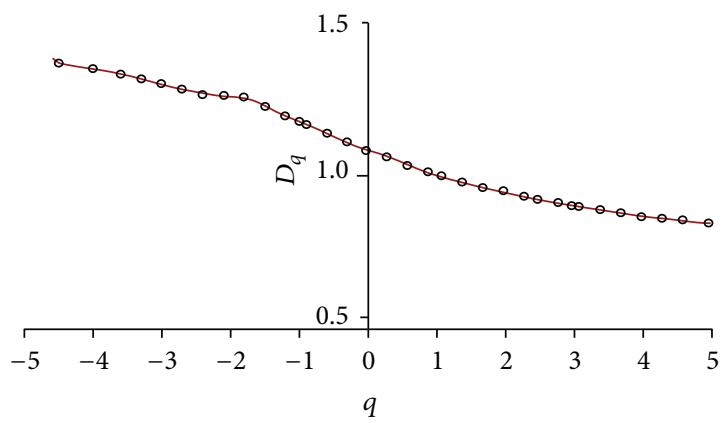

(c)

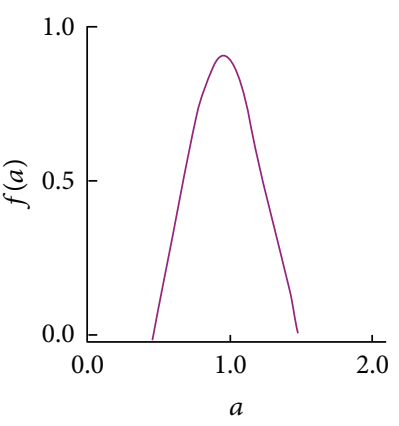

(d)

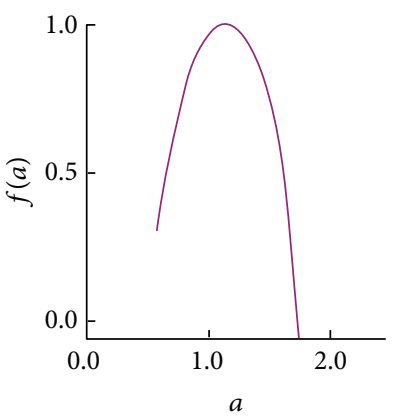

(e)

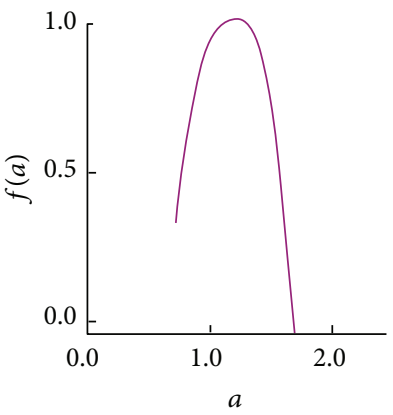

(f)

FIGURE 1: The three variation stages of the generalized dimension spectrum and singularity spectrum before 22 April 1974 Liyang earthquake (M5.5). (a) and (d) show the generalized dimension spectrum and singularity spectrum two years prior to this earthquake, respectively; (b) and (e) show the generalized dimension spectrum and singularity spectrum seven months prior to this earthquake, respectively; (c) and (f) show the generalized dimension spectrum and singularity spectrum four months prior to this earthquake, respectively. The curve of the generalized dimension spectrum in Figure 1(b) is steeper than the two others, and the value range of the singularity spectrum in Figure 1(e) is broader than the two others.

variation stage of the generalized dimension spectrum and singularity spectrum (four months prior to this earthquake), respectively, in which the level difference between the left end and right end of the generalized dimension spectrum becomes small again. Figure 2 shows the three variation stages of the generalized dimension spectrum and singularity spectrum before 9 July 1979 Liyang M6.0 earthquake (the data set includes 827 earthquakes). The time in which the anomalous change appeared is fifteen months before this earthquake (see anomalous change curves in Figures 2(b) and 2(e)). Figure 3 shows the three variation stages of the generalized dimension spectrum and singularity spectrum before and after 21 May 1984 Wunansha M6.2 earthquake (the data set includes 937 earthquakes). The time in which the anomalous change appeared is eight months before this earthquake (see anomalous change curves in Figures 3(b) and 3(e)). Figure 4 shows the three variation stages of the generalized dimension spectrum and singularity spectrum before 9 November 1996 Yellow Sea M6.1 earthquake (the data set includes 679 earthquakes). The time in which the anomalous change appeared is seventeen months before this earthquake (see anomalous change curves in Figures 4(b) and $4(\mathrm{e})$ ). According to the previously mentioned anomalous changes of the generalized dimension spectrum and singularity spectrum in the studied cases, I believe that it is significant to apply the multifractal characteristics to midstrong earthquake forecasting in this area.

Kiyashchenko et al. [16] studied the changes in the abscissa of the top of multifractal spectrum (i.e., the singularity spectrum) by carrying out the simulation of the crack 


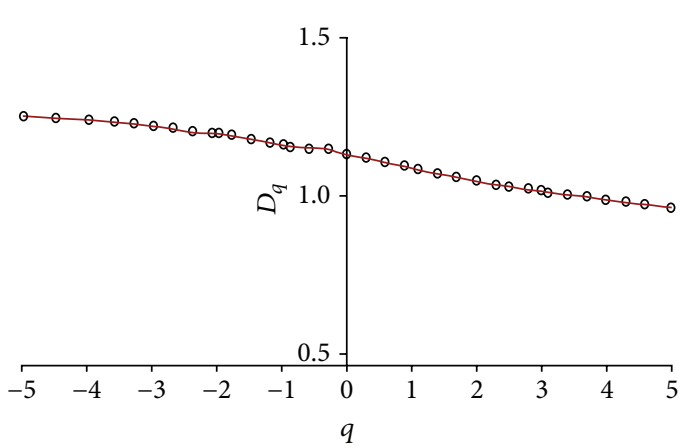

(a)

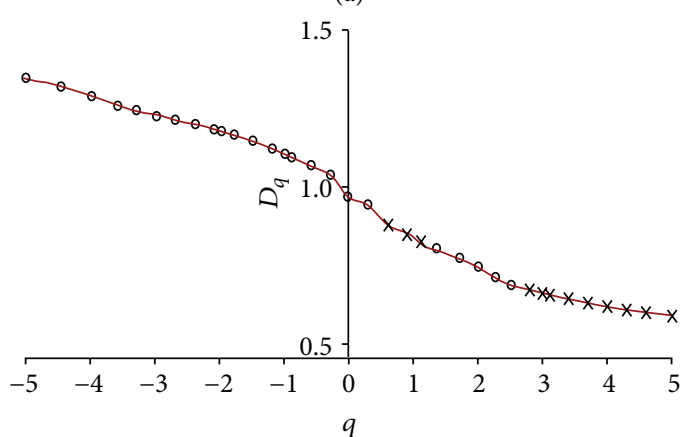

(b)

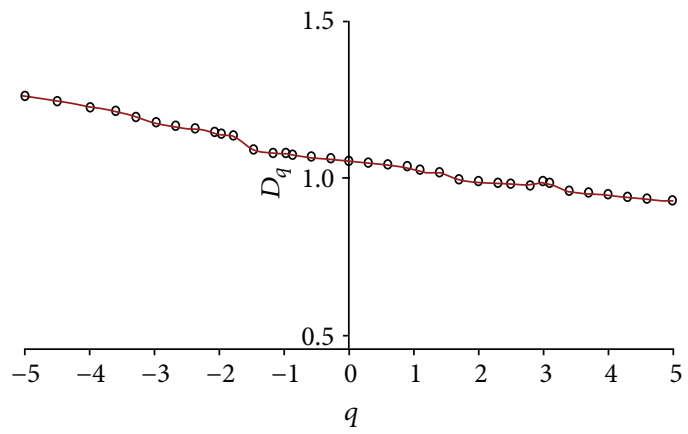

(c)

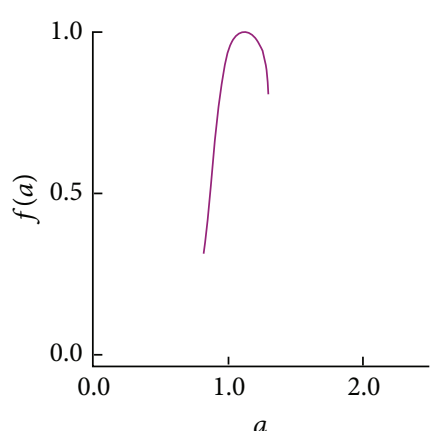

(d)

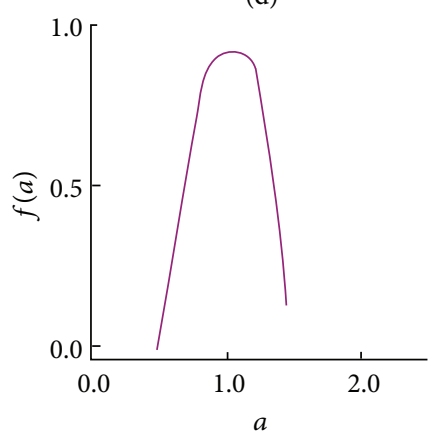

(e)

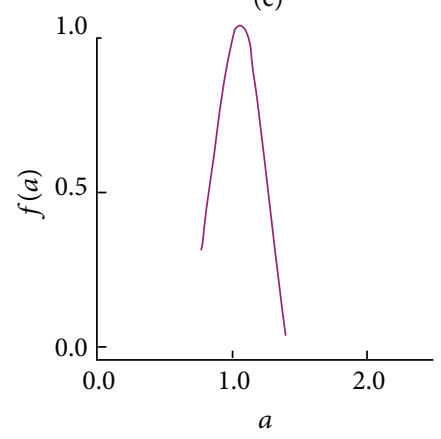

(f)

FIGURE 2: The three variation stages of the generalized dimension spectrum and singularity spectrum before 9 July 1979 Liyang earthquake (M6.0). (a) and (d) show the generalized dimension spectrum and singularity spectrum two years prior to this earthquake, respectively; (b) and (e) show the generalized dimension spectrum and singularity spectrum fifteen months prior to this earthquake, respectively; (c) and (f) show the generalized dimension spectrum and singularity spectrum eight months prior to this earthquake, respectively. The curve of the generalized dimension spectrum in Figure 2(b) is steeper than the two others, and the value range of the singularity spectrum in Figure 2(e) is broader than the two others.

network evolution in elastic body forced by outside forces. They found that the changes in the abscissa of the top of multifractal spectrum contain some useful information of the development of the system towards main fracture. Their simulation results were also upheld by the studies on some instances of the seismicity in Japan and Southern California.

The characteristic changes of multifractal spectrum of seismicity have been studied for many years. The observed cases in which there are characteristic changes of multifractal spectrum of seismicity prior to large earthquakes are not few. The advantage of the multifractal spectrum is that it can sufficiently give the description of heterogeneous properties of a fractal system. However, it is not good at presenting the property of self-affine fractal and is incapable to describe local features of the fractal seismic system because it can only provide the description of global properties of fractal objects.

\section{Correlation Dimension and Fractal Dimension}

The calculation of correlation dimension $D_{2}$ is the special circumstance of generalized dimension calculation. If we allocate $q=2$ in (1) and (2), the correlation dimension $D_{2}$ can be obtained.

The fractal dimension $D$ of seismicity can be computed by applying the correlation integral algorithm [38, 42]. 

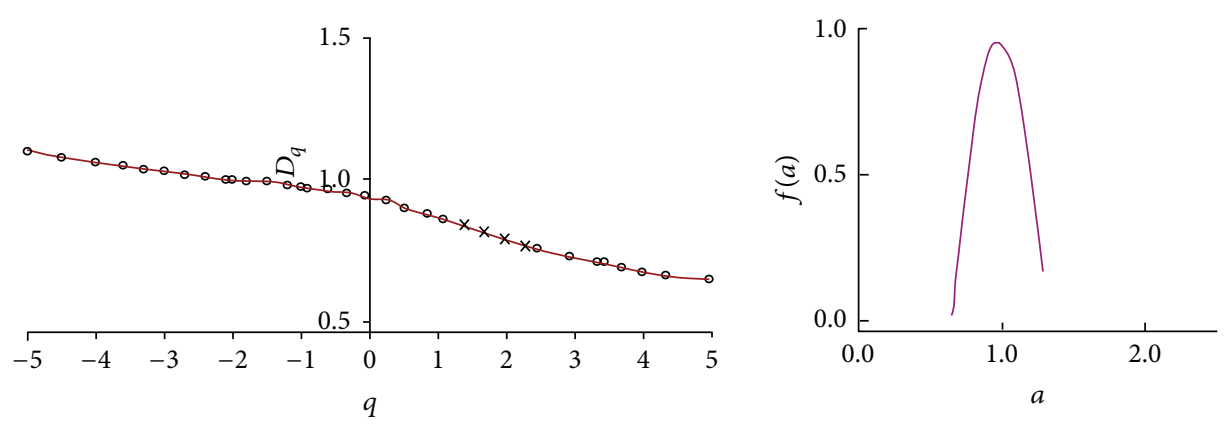

(a)

(d)
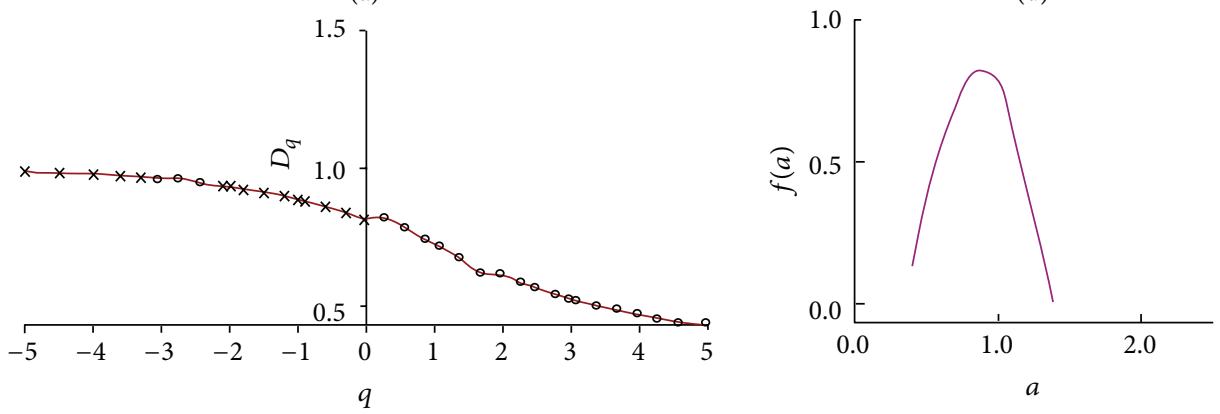

(b)

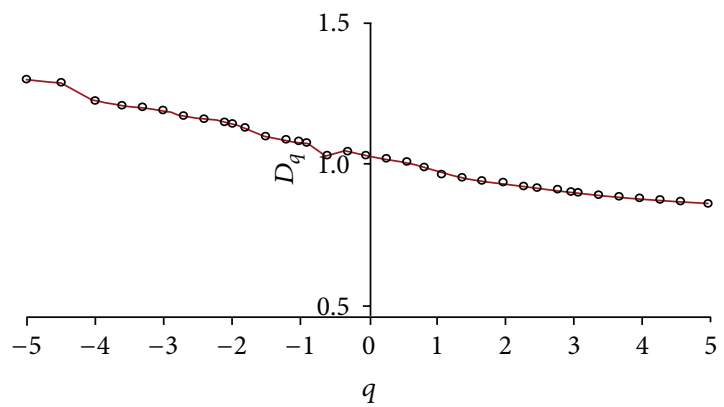

(e)

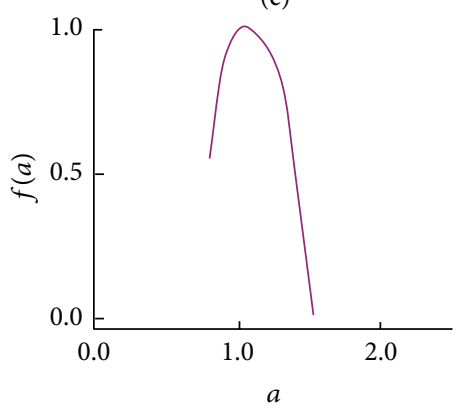

(c)

(f)

FIGURE 3: The three variation stages of the generalized dimension spectrum and singularity spectrum before and after 21 May 1984 Wunansha earthquake (M6.2). (a) and (d) show the generalized dimension spectrum and singularity spectrum two years prior to this earthquake, respectively; (b) and (e) show the generalized dimension spectrum and singularity spectrum eight months prior to this earthquake, respectively; (c) and (f) show the generalized dimension spectrum and singularity spectrum ten months after this earthquake, respectively. The curve of the generalized dimension spectrum in Figure 3(b) is steeper than the two others, and the value range of the singularity spectrum in Figure 3(e) is broader than the two others.

The correlation integral $C(r)$ is expressed by the following formula [21]:

$$
C(r)=\frac{2 N(R<r)}{N(N-1)}
$$

Here, $N$ is the number of data points used for analysis, $N(R<$ $r$ ) is the quantity of data points within a distance $R$ that is less than $r$. If the seismic distribution has the property of fractals, the following relational expression can be got:

$$
C(r) \propto r^{D}
$$

The $D$ in the relational expression is defined as the fractal dimension [38].

Lei and Satoh [24] studied the statistic characteristic of prefailure harm on the basis of acoustic emission events
(AE) observed through the whole course of the great fracture of representative rock samples under dissimilar compression. They found that the prefailure harm evolution before great fracture in several representative rocks is typified by a reduced correlation dimension. They believed that this precursory change in correlation dimension may promote short-term prediction for the critical point behavior prior to rock failure.

Lu et al. [25] studied the change in the fractal dimension during the process of a damage development, especially during the process of impending critical failure. They found that the fractal dimension of the spatial distribution of microcracks reduces as the damage develops. Their conclusion is that an abrupt decrease in fractal dimension can be served as an indicator of a possible precursor which presages an impending catastrophic rupture. 


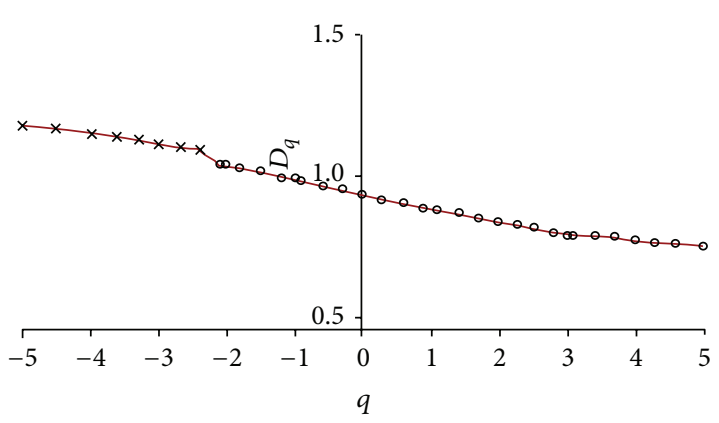

(a)

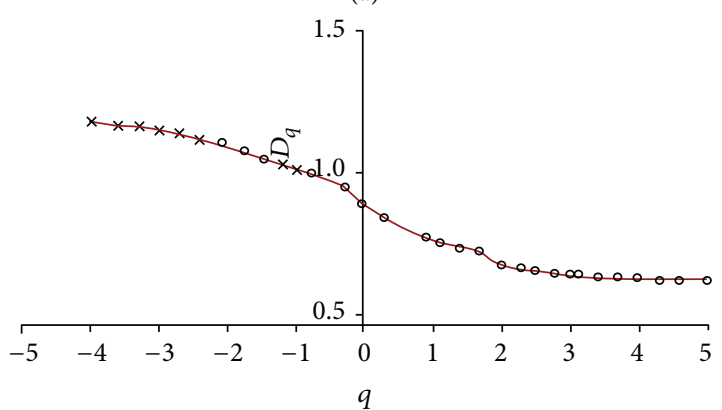

(b)

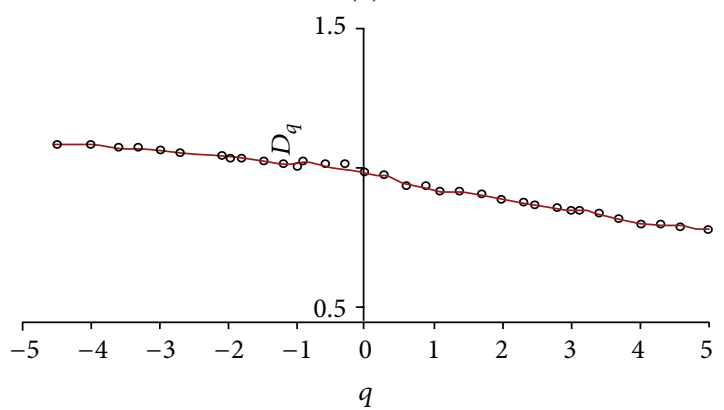

(c)

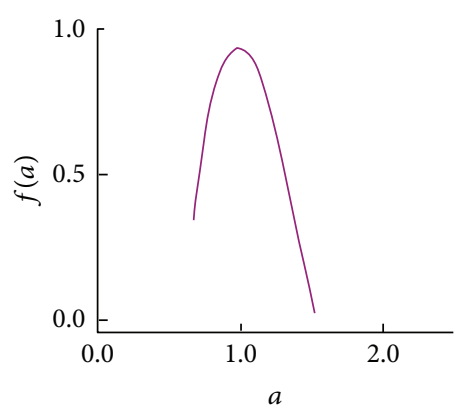

(d)

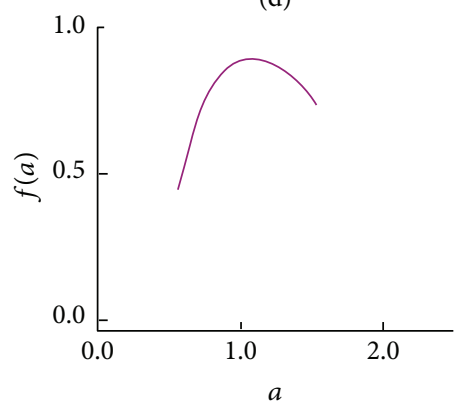

(e)

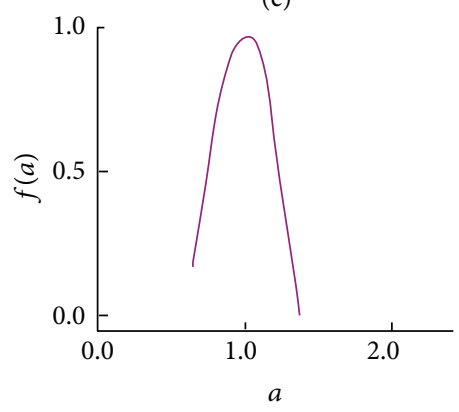

(f)

FiguRE 4: The three variation stages of the generalized dimension spectrum and singularity spectrum before 9 November 1996 Yellow Sea earthquake (M6.1). (a) and (d) show the generalized dimension spectrum and singularity spectrum two years prior to this earthquake, respectively; (b) and (e) show the generalized dimension spectrum and singularity spectrum seventeen months prior to this earthquake, respectively; (c) and (f) show the generalized dimension spectrum and singularity spectrum five months before this earthquake, respectively. The curve of the generalized dimension spectrum in Figure 4(b) is steeper than the two others, and the value range of the singularity spectrum in Figure 4(e) is broader than the two others.

Roy and Nath [22] studied the variation of the correlation dimension before and after the Great Sumatra earthquake of 26 December $2004\left(M_{w}=9\right)$ by analyzing the seismic events $(m(b) \geq 4)$ occurring in the period of 1996-2005 in Sumatra region. They found that there is a decrease of correlation dimension in the short time period before this earthquake. Similar precursory changes were also found ahead of the Izmith earthquake of 17 August $1999\left(M_{s}=7.8\right)$ and the 3 November 2002 Dennali earthquake of 3 November 2002 $\left(M_{s}=8.5\right)$.

Roy and Padhi [19] also studied the correlation dimension of seismicity in Iran and neighbouring areas. They found that the precursory clustering pattern in the short time period before three large earthquakes $\left(M_{w}=7.8\right.$ on 16.9.1978; $M_{w}=$ 6.8 on 26.12.2003; $M_{w}=7.7$ on 10.5.97) can be recognized by analyzing the change in the correlation dimension of seismicity in this area. Their conclusion is that the probability for future large earthquakes can be evaluated by studying correlation dimension of seismicity which is related to the temporal and spatial clustering pattern of earthquakes.

Enescu and Ito [43] studied the changes of the correlation dimension in a large region near the epicenter of the 1995 Hyogo-ken Nanbu (Kobe) earthquake and in the focal region. They found that the anomalous changes of the correlation dimension turn up about two years prior to the occurrence of the 1995 Hyogo-Ken Nanbu (Kobe) earthquake, and these anomalous changes turn up in a relatively broad area near the epicenter of the 1995 Hyogo-ken Nanbu (Kobe) earthquake and in the focal region.

Murase [21] studied the variations of the pattern of hypocenter distribution before the 2003 Tokachi-oki earthquake $\left(M_{J}=8.0\right)$ by analyzing the temporal change 
on the spatial fractal dimension. He found that the value of fractal dimension began to reduce in 1998 and had kept its low value for about a year prior to the occurrence of the previous great earthquake. He believes that the reduction of fractal dimension prior to the main shock is a typical feature of some large earthquakes and may be considered as a precursor of the large earthquakes. His conclusion is that it is beneficial to capture precursory information of seismicity prior to a large earthquake by analyzing the temporal change of fractal dimension.

Kiyashchenko et al. [16] analyzed the changes of fractal dimension in a study in which they carried out the simulation of the crack network evolution in elastomer acted on by outside forces. They found that the crack network develops from the condition in which the distributions of cracks and seismicity are random and chaotic to the state in which the patterns are fractal and clustered. Not only that, the study results of some instances of the seismicity in Japan and Southern California upheld the simulation results. Their conclusion is that the changes on fractal dimensions comprise some useful information on the development of the system towards main fracture.

Teotia and Kumar [20] studied the correlation dimension by analyzing the seismic catalog data for the period of 1973-2009 containing the Muzaffarabad-Kashmir earthquake $\left(M_{w}=7.6\right)$ of October 2005 in the North-Western Himalaya area. They found the significant temporal variations of correlation dimension before the occurrence of Muzaffarabad-Kashmir earthquake in relation to epicenter distribution in the area. They concluded that the observed change of the correlation dimension prior to the large earthquake may help us to recognize the seismogenic zone of large seismic events in different tectonic areas.

So far, there have been many studied cases ranging from petrophysical experiments, rock burst to large natural earthquakes, in which the correlation dimension or fractal dimension decreased before the main raptures. Therefore, the research in this respect is relatively mature. However, because the method of correlation dimension and fractal dimension can only describe the monofractal property of seismicity, it is inefficient to describe the heterogeneous property of the seismicity. Thus, the useful information about heterogeneous property of fractal seismic system will be lost when we use these methods.

\section{4. $R / S$ Hurst Analysis}

The method of $R / S$ Hurst analysis is introduced by Hurst who developed this method to aim at studying the flow of the Nile River [44]. Yet, this method is also applied to analyze observed time series including the interevent time series of the seismic sequences [27] and the time series of earthquake frequency $[30,45]$.

The method of $R / S$ Hurst analysis is as follows [46, 47].

Suppose that a typical time series is expressed by $\xi(t)(t=$ $1,2, \ldots)$. For a positive integer $\tau$, the average value of $\xi(t)$ is

$$
\langle\xi\rangle_{\tau}=\frac{1}{\tau} \sum_{t=1}^{\tau} \xi(t) .
$$

The accumulated deviation $X(t, \tau)$ is expressed as

$$
X(t, \tau)=\sum_{u=1}^{t}\left[\xi(u)-\langle\xi\rangle_{\tau}\right] \quad 1 \leq t \leq \tau .
$$

Corresponding to the time $t$ that varies from 1 to $\tau$, the values of accumulated deviation can be obtained. The difference between the maximal accumulated deviation and the minimal deviation is called as range $R(\tau)$, and it is expressed by

$$
R(\tau)=\max _{1 \leq t \leq \tau} X(t, \tau)-\min _{1 \leq t \leq \tau} X(t, \tau) .
$$

The range $R(\tau)$ value is the maximal fluctuation of the accumulated deviation in the time interval $1 \leq t \leq \tau$. The standard deviation $S(\tau)$ in the same time interval is defined as

$$
S(\tau)=\left[\frac{1}{\tau} \sum_{t=1}^{\tau}\left(\xi(t)-\langle\xi\rangle_{\tau}\right)^{2}\right]^{1 / 2} .
$$

For the dissimilar time length $\tau$, the dimensionless value $R / S$ is represented by the following empirical relationship:

$$
\frac{R(\tau)}{S(\tau)} \propto \tau^{H} .
$$

Equation (9) is called as the $R / S$ empirical relational expression, which shows that the ratio of range to standard deviation changes with the power of time length. The exponent $H$ is defined Hurst exponent that ranges from 0 to 1 .

Zhao and Wang [27] studied the temporal variation of Hurst exponent for the sequence of the interval time between earthquakes several years before and after some large earthquakes $\left(M_{s} \geq 7.0\right)$ in China Mainland since 1970. They found that the anomalous change of decrease of Hurst exponent appeared two years or so before the large earthquakes. They believe that this variation feature of anomaly of Hurst exponent can be considered as a medium-short-term earthquake precursor, and the anomalous process of Hurst exponent reveals the property of seismicity from disorder to order in a large earthquake generating system.

Guo [30] studied the temporal variation of Hurst exponent for the earthquake frequency before some medium and strong earthquakes (magnitudes range from M5.0 to 7.8) in north China. He found that there is an anomalous characteristic that the value of Hurst exponent is less than 0.87 one or two years before some earthquakes, and the anomalous time of Hurst exponent ranges from three months to two years. He believes that anomalous characteristic of Hurst exponent for earthquake frequency can be served as a medium-short-term indicator for the medium and strong earthquake forecasting.

$\mathrm{Li}$ and Wang [48] studied the temporal variation of Hurst exponent for the earthquake frequency before and after twelve strong earthquakes (magnitudes range from M5.7 to 6.6) in Qinghai-Tibet Plateau in China. They found that the value of Hurst exponent for seven earthquake cases decreases before the occurrence of the strong earthquakes, and this decrease continues more than three months. He believes that such anomalous change of Hurst exponent for earthquake frequency is related to seismicity in Qinghai-Tibet Plateau. 


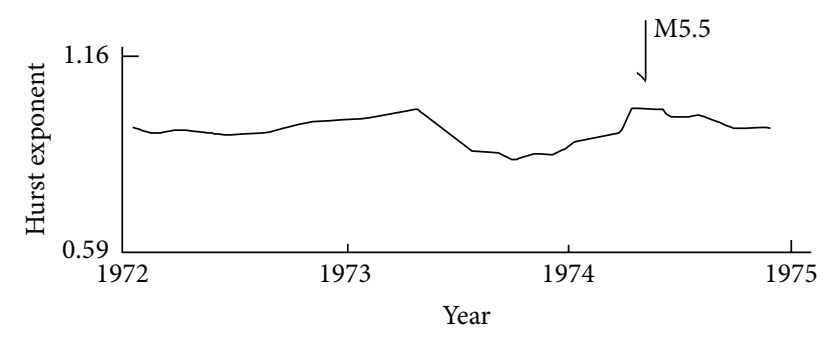

FIGURE 5: The temporal variation of Hurst exponent before and after the 22 April 1974 Liyang earthquake (M5.5).

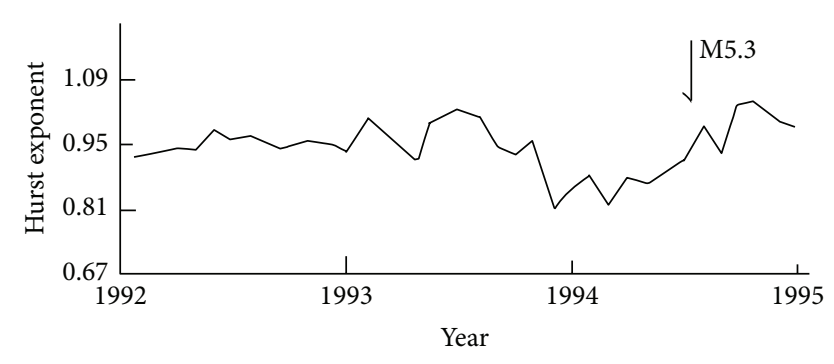

FIGURE 6: The temporal variation of Hurst exponent before and after the 26 July 1994 Yellow Sea earthquake (M5.3).

Wang et al. [49] studied the temporal change of Hurst exponent for the earthquake frequency before and after fourteen earthquakes $\left(M_{s} \geq 7.2\right)$ in China Mainland. They found that the decrease changes of Hurst exponent for most of earthquake cases (about 78 percent of the earthquake cases) appeared several months to one and half years before the occurrence of the strong earthquakes. He believes that such decrease change of Hurst exponent for earthquake frequency is meaningful for the earthquake forecasting.

$\mathrm{Li}$ and $\mathrm{Xu}$ [45] studied the temporal variation of Hurst exponent for earthquake frequency by analyzing the seismicity data in Jiangsu and adjacent area, where several midstrong earthquakes (magnitudes ranging from M5.0 to 6.2) occurred. We found that the anomalous changes in the Hurst exponent for some earthquake cases appear several months to about a little over a year before the mid-strong earthquakes. Figure 5 shows the temporal variation of Hurst exponent before and after the 22 April 1974 Liyang earthquake (M5.5). As can be seen from Figure 5, the Hurst exponent fluctuates within small range and shows no feature change before March 1973. However, it begins to decrease in March 1973. After Liyang earthquake, it increases back to the state of fluctuating before March 1973. Figure 6 shows the temporal variation of Hurst exponent before and after the 26 July 1994 Yellow Sea earthquake (M5.3). From Figure 6 we see that the Hurst exponent fluctuates within small range before September 1993. The Hurst exponent begins to decrease on September 1993. After Yellow Sea earthquake, it gradually returns to the level before September 1993. Figure 7 shows the temporal variation of Hurst exponent before and after the 10 February 1990 Changshu earthquake (M5.1). As can be seen from Figure 7, the Hurst exponent fluctuates within small limit and shows no characteristic variation before March 1989.

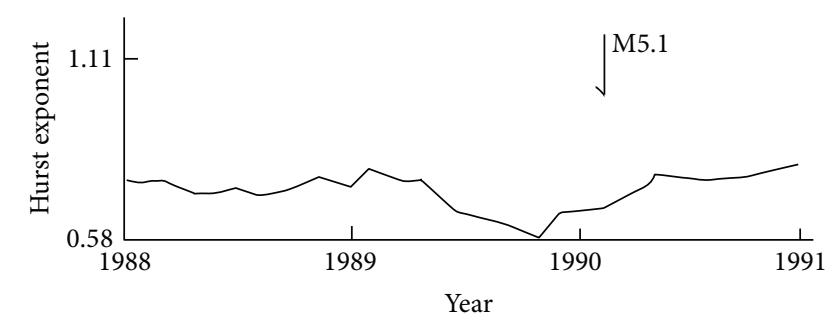

FIGURE 7: The temporal variation of Hurst exponent before and after the 10 February 1990 Changshu earthquake (M5.1).

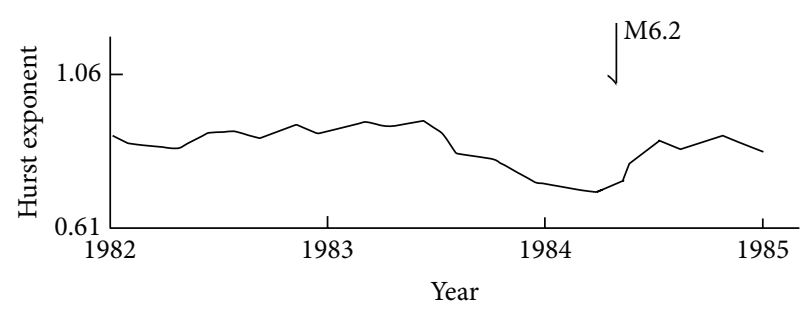

FIGURE 8: The temporal variation of Hurst exponent before and after the 21 May 1984 Wunansha earthquake (M6.2).

The Hurst exponent begins to decrease in March 1989. After Changshu earthquake, it increases gradually back to the state of fluctuating before March 1989. Figure 8 shows the temporal variation of Hurst exponent before and after the 21 May 1984 Wunansha earthquake (M6.2). From Figure 8 we see that the Hurst exponent fluctuates within small range before June 1983. The Hurst exponent begins to decrease on June 1983. After Wunansha earthquake, it quickly returns to the level before June 1983. Based on our study, we believe that these anomalous changes in the Hurst exponent can be of reference significance in earthquake forecasting in this area.

Because the Hurst exponent analysis is good at presenting the property of self-affine fractal of seismicity, it is efficient for analyzing temporal characteristic variation of self-affine fractal of seismicity prior to large earthquakes. However, the Hurst exponent analysis is incapable to describe the heterogeneous property of the fractal objects. Thus, the heterogeneous characteristic of the seismicity will not be demonstrated if we use this method.

\section{Local Scaling Property of Seismicity}

The method of local scaling property is a typical method that focuses on the local property of fractal bodies. It is as follows [50-52].

A particular characteristic of fractal bodies is that they have the feature of asymptotical self-similarity at small length scales. Assume that a fractal is represented by a real function $f$. Viewing near a discretionary point $x_{0}$ of function $f$ at different scales, we can invariably observe the similar function up to a scaling factor. Letting

$$
f_{x_{0}}(x)=f\left(x_{0}+x\right)-f\left(x_{0}\right),
$$


we obtain

$$
f_{x_{0}}(\lambda x)=\lambda^{+\alpha\left(x_{0}\right)} f_{x_{0}}(x) .
$$

Here, $\alpha\left(x_{0}\right)$ is defined as the local scaling exponent (also called the singularity exponent), indicating the singularity strength at point $x_{0}$.

It may be confirmed that the wavelet transform coefficient of $f(x)$ close to the point $x_{0}$ shows the same property of scale invariance as well. We treat

$$
T\left(a, x_{0}+b\right)=\frac{1}{\sqrt{a}} \int f(x) \psi\left(\frac{x-x_{0}-b}{a}\right) d x
$$

as the wavelet transform close to the point $x_{0}$ and

$$
T\left(\lambda a, x_{0}, \lambda b\right)=\frac{1}{\sqrt{\lambda a}} \int f(x) \psi\left(\frac{x-x_{0}-\lambda b}{\lambda a}\right) d x
$$

as the form of the wavelet transform coefficient close to the point $x_{0}$ when the scale varies. This transformation can be served as a mathematical microscope, the position and magnification of which are $b$ and $l / a$, respectively, and the optics of which are determined by the selection of the specific wavelet $\psi$. Then, we obtain

$$
T\left(\lambda a, x_{0}+\lambda b\right)=\lambda^{+\alpha\left(x_{0}\right)+1 / 2} T\left(a, x_{0}+b\right) .
$$

Corresponding to the different positions $b$, where the fractal is asymptotically self-similar at small scales, the plot of $\ln |T(a, b)|$ versus $\ln a$ shows an approximate straight line whose slope $K$ can be obtained by using a least squares fit with a check value of $F$ test. Thus, the local scaling exponent $\alpha$ at position $b$ can be got from the following expression:

$$
K=\alpha+\frac{1}{2} .
$$

Changing parameter $b$, the relationship between the position and the local scaling exponent $\alpha$ can be got.

In the practical process of calculation, it is necessary to select suitable wavelet transform parameters which correspond to the seismicity features of different regions by using trial-and-error method. The plots of $\ln |T(a, b)|$ versus $\ln a$, which we get by calculating the series of interevent times between successive earthquakes point by point, show two dissimilar types: one is the proximate straight line, the other is the fluctuating line. For the proximate straight line, the slope $K$ can be got by performing the computation of least square fit with the help of test value (if there are the oscillations which attach to the proximate straight line, the fitting calculation should be performed after the oscillations are deleted for the purpose of avoiding the erroneous fitting result.). Therefore, the local scaling exponent $\alpha$ at such points can be calculated using (15), and such points are defined as singular points. For the fluctuating line, both the slope $K$ and local scaling exponent $\alpha$ cannot be obtained by doing fit, which means that singularity does not exist at these points. Thus, by analyzing temporal distribution features of singular points, we can obtain the temporal variation of local scaling property of seismicity.
$\mathrm{Li}$ and $\mathrm{Xu}[26,39-41]$ studied the temporal variation of local scaling property for the series of interevent times between successive earthquakes by using the seismic activity data in several regions of China. We found that there are characteristic changes of local scaling property prior to some large earthquakes. We believe that such characteristic changes of local scaling property might be useful for us to get precursory information about the scale invariance of seismicity before large earthquakes.

The strengths of the method of local scaling property are that it cannot only give the description of heterogeneous properties of seismicity but also give the description of the local features of it. However, there are also some weaknesses in this method. Firstly, because the calculation of this method is done point by point and the process of calculation is complex, thus, if we do not control the cumulative error in the calculation process, the deviation of calculation results will be large. Secondly, it is the complicated process to choose suitable wavelet transform parameters which correspond to the seismicity features of different regions by using trialand-error method. If we do not choose suitable parameters, the useful information before large earthquakes will be concealed. Thirdly, the observed cases in which there are characteristic changes of local scaling property of seismicity prior to large earthquakes have been quite few, and the research results have not widely been examined so far. Therefore, the research results obtained are only tentative, and cannot be treated as the final results. Nevertheless, the study on the characteristic changes of local scaling property of seismicity is meaningful, because it is carried out from a new theoretical viewpoint.

\section{Conclusions}

In this paper, we presented some important research developments of characteristic changes of scale invariance of seismicity before large earthquakes. These studied cases are of particular importance to the researchers in earthquake forecasting and seismic activity. Meanwhile, the strengths and weaknesses of different methods of scale invariance are discussed. There have been many studied cases in which there are characteristic changes of the multifractal spectrum, the correlation dimension, the fractal dimension, and Hurst exponent analysis of seismicity prior to large earthquakes, indicating that the research results obtained by using the multifractal spectrum, the correlation dimension, the fractal dimension, and Hurst exponent analysis are relatively mature, while there have only been few observed cases in which there are the characteristic changes of local scaling property of seismicity prior to large earthquakes, indicating that the research results obtained by using the method of local scaling property are tentative and not final. The advantage of the method of multifractal spectrum is that it can sufficiently give the description of heterogeneous properties of a fractal system, but, it is not good at presenting the property of self-affine fractal and is incapable to describe local features of the fractal seismic system. The peculiarity of the correlation dimension and fractal dimension is that they can only describe the monofractal property of seismicity. The advantage of the 
Hurst exponent analysis is that it is good at presenting the property of self-affine fractal of seismicity; however, it is incapable to describe the heterogeneous property of the fractal objects. The peculiarity of local scaling property is that it can not only give the description of heterogeneous properties of seismicity but also give the description of the local features of it.

Due to the previously mentioned strengths and weaknesses of different methods of scale invariance, we suggest that when people try to obtain the precursory information before large earthquakes or to study the fractal property of seismicity by means of the previous scale invariance methods, the strengths and weaknesses of these methods have to be taken into consideration for the purpose of increasing research efficiency. If they do not consider the strengths and weaknesses of these methods, the efficiency of their research might greatly decrease.

\section{Acknowledgments}

The authors thank Professor S. S. Dong for helpful conversation. This work is supported by the Natural Science Foundation of Jiangsu province, China (BK2008486).

\section{References}

[1] T. Chelidze and T. Matcharashvili, "Complexity of seismic process; measuring and applications-a review," Tectonophysics, vol. 431, no. 1-4, pp. 49-60, 2007.

[2] B. Enescu, K. Ito, M. Radulian, E. Popescu, and O. Bazacliu, "Multifractal and chaotic analysis of Vrancea (Romania) intermediate-depth earthquakes: investigation of the temporal distribution of events," Pure and Applied Geophysics, vol. 162, no. 2, pp. 249-271, 2005.

[3] Y. Y. Kagan, "Earthquake spatial distribution: the correlation dimension," Geophysical Journal International, vol. 168, no. 3, pp. 1175-1194, 2007.

[4] D. Kiyashchenko, N. Smirnova, V. Troyan, and F. Vallianatos, "Dynamics of multifractal and correlation characteristics of the spatio-temporal distribution of regional seismicity before the strong earthquakes," Natural Hazards and Earth System Science, vol. 3, no. 3-4, pp. 285-298, 2003.

[5] R. J. Mittag, "Fractal analysis of earthquake swarms of Vogtland/ NW-Bohemia intraplate seismicity," Journal of Geodynamics, vol. 35, no. 1-2, pp. 173-189, 2003.

[6] A. O. Oncel and T. H. Wilson, "Space-time correlations of seismotectonic parameters: examples from Japan and from Turkey preceding the İzmit earthquake," Bulletin of the Seismological Society of America, vol. 92, no. 1, pp. 339-349, 2002.

[7] L. Telesca, G. Hloupis, I. Nikolintaga, and F. Vallianatos, "Temporal patterns in southern Aegean seismicity revealed by the multiresolution wavelet analysis," Communications in Nonlinear Science and Numerical Simulation, vol. 12, no. 8, pp. 1418-1426, 2007.

[8] V. Uritsky, N. Smirnova, V. Troyan, and F. Vallianatos, "Critical dynamics of fractal fault systems and its role in the generation of pre-seismic electromagnetic emissions," Physics and Chemistry of the Earth, vol. 29, no. 4-9, pp. 473-480, 2004.

[9] F. Vallianatos, G. Michas, G. Papadakis, and P. Sammonds, "A non-extensive statistical physics view to the spatiotemporal properties of the June 1995, Aigion earthquake (M6.2) aftershock sequence (West Corinth rift, Greece)," Acta Geophysica, vol. 60, no. 3, pp. 758-768, 2012.

[10] F. Vallianatos, G. Michas, G. Papadakis, and A. Tzanis, "Evidence of non-extensivity in the seismicity observed during the 2011-2012 unrest at the Santorini volcanic complex, Greece," Natural Hazards and Earth System Sciences, vol. 13, pp. 177-185.

[11] F. Vallianatos, A. Nardi, R. Carluccio, and M. Chiappini, "Experimental evidence of a non-extensive statistical physics behavior of electromagnetic signals emitted from rocks under stress up to fracture. Preliminary results," Acta Geophysica, vol. 60, no. 3, pp. 894-909, 2012.

[12] F. Vallianatos and A. Tzanis, "On the nature, scaling and spectral properties of pre-seismic ULF signals," Natural Hazards and Earth System Science, vol. 3, no. 3-4, pp. 237-242, 2003.

[13] A. Zamani and M. Agh-Atabai, "Temporal characteristics of seismicity in the Alborz and Zagros regions of Iran, using a multifractal approach," Journal of Geodynamics, vol. 47, no. 5, pp. 271-279, 2009.

[14] F. Caruso, S. Vinciguerra, V. Latora, A. Rapisarda, and S. Malone, "Multifractal analysis of Mount St. Helens seismicity as a tool for identifying eruptive activity," Fractals, vol. 14, no. 3, pp. 179-186, 2006.

[15] P. P. Dimitriu, E. M. Scordilis, and V. G. Karacostas, "Multifractal analysis of the Arnea, Greece Seismicity with potential implications for earthquake prediction," Natural Hazards, vol. 21, no. 2-3, pp. 277-295, 2000.

[16] D. Kiyashchenko, N. Smirnova, V. Troyan, E. Saenger, and F. Vallianatos, "Seismic hazard precursory evolution: fractal and multifractal aspects," Physics and Chemistry of the Earth, vol. 29, no. 4-9, pp. 367-378, 2004.

[17] Q. Li, "The multifractal characteristics of the seismic temporal series in Jiangsu Province and adjacent areas and their application to earthquake prediction," Journal of Seismological Research, vol. 25, pp. 257-261, 2002 (Chinese).

[18] S. Nakaya, "Fractal properties of seismicity in regions affected by large, shallow earthquakes in western Japan: implications for fault formation processes based on a binary fractal fracture network model," Journal of Geophysical Research B, vol. 110, no. 1, Article ID B01310, 2005.

[19] P. N. S. Roy and A. Padhi, "Multifractal analysis of earthquakes in the Southeastern Iran-Bam Region," Pure and Applied Geophysics, vol. 164, no. 11, pp. 2271-2290, 2007.

[20] S. S. Teotia and D. Kumar, "Role of multifractal analysis in understanding the preparation zone for large size earthquake in the North-Western Himalaya region," Nonlinear Processes in Geophysics, vol. 18, no. 1, pp. 111-118, 2011.

[21] K. Murase, "A characteristic change in fractal dimension prior to the 2003 Tokachi-oki Earthquake $(M J=8.0)$, Hokkaido, Northern Japan," Earth, Planets and Space, vol. 56, no. 3, pp. 401-405, 2004.

[22] P. N. S. Roy and S. K. Nath, "Precursory correlation dimensions for three great earthquakes," Current Science, vol. 93, no. 11, pp. 1522-1529, 2007.

[23] X. L. Lei, K. Kusunose, T. Satoh, and O. Nishizawa, "The hierarchical rupture process of a fault: an experimental study," Physics of the Earth and Planetary Interiors, vol. 137, no. 1-4, pp. 213-228, 2003.

[24] X. L. Lei and T. Satoh, "Indicators of critical point behavior prior to rock failure inferred from pre-failure damage," Tectonophysics, vol. 431, no. 1-4, pp. 97-111, 2007. 
[25] C. Lu, Y. W. Mai, and H. Xie, "A sudden drop of fractal dimension: a likely precursor of catastrophic failure in disordered media," Philosophical Magazine Letters, vol. 85, no. 1, pp. 33-40, 2005.

[26] Q. Li and G. M. Xu, "Relationship between the characteristc variations of local scaling property and the process of seismogeny: the revelation of a new physical mechanism of seismicity," Fractals, vol. 18, no. 2, pp. 197-206, 2010.

[27] C. P. Zhao and H. T. Wang, "Anomalous features of Hurst exponent before some large earthquakes in China Mainland," Inland Earthquake, vol. 15, pp. 331-337, 2001 (Chinese).

[28] A. Carpinteri, G. Lacidogna, and S. Puzzi, "From criticality to final collapse: evolution of the "b-value" from 1.5 to 1.0," Chaos, Solitons and Fractals, vol. 41, no. 2, pp. 843-853, 2009.

[29] C. Goltz, Fractal and Chaotic Properties of Earthquakes, Springer, Berlin, Germany, 1997.

[30] D. K. Guo, "Application of Hurst exponent of earthquake frequency to the earthquake prediction," North China Earthquake Sciences, vol. 20, pp. 44-50, 2002 (Chinese).

[31] Y. T. Lee, C. C. Chen, Y. F. Chang, and L. Y. Chiao, "Precursory phenomena associated with large avalanches in the long-range connective sandpile (LRCS) model," Physica A, vol. 387, no. 21, pp. 5263-5270, 2008.

[32] T. Matcharashvili, T. Chelidze, and Z. Javakhishvili, "Nonlinear analysis of magnitude and interevent time interval sequences for earthquakes of the Caucasian region," Nonlinear Processes in Geophysics, vol. 7, no. 1-2, pp. 9-19, 2000.

[33] M. Radulian and C. I. Trifu, "Would it have been possible to predict the 30 August 1986 Vrancea earthquake?" BulletinSeismological Society of America, vol. 81, no. 6, pp. 2498-2503, 1991.

[34] L. Telesca and V. Lapenna, "Measuring multifractality in seismic sequences," Tectonophysics, vol. 423, no. 1-4, pp. 115-123, 2006.

[35] L. Telesca, V. Lapenna, and M. MacChiato, "Multifractal fluctuations in seismic interspike series," Physica A, vol. 354, no. 1-4, pp. 629-640, 2005.

[36] P. Grassberger and I. Procaccia, "Dimensions and entropies of strange attractors from a fluctuating dynamics approach," Physica D, vol. 13, no. 1-2, pp. 34-54, 1984.

[37] A. Chhabra and R. V. Jensen, "Direct determination of the singularity spectrum," Physical Review Letters, vol. 62, no. 12, pp. 1327-1330, 1989.

[38] P. Grassberger, "Generalized dimensions of strange attractors," Physics Letters A, vol. 97, no. 6, pp. 227-230, 1983.

[39] Q. Li and G. M. Xu, "Local scaling property of seismicity: an example of getting valuable information from complex hierarchical system," Nonlinear Processes in Geophysics, vol. 17, no. 5, pp. 423-429, 2010.

[40] Q. Li and G. M. Xu, "Characteristic variation of local scaling property before Puer M6.4 earthquake in China: the presence of a new pattern of nonlinear behavior of seismicity," Izvestiya, Physics of the Solid Earth, vol. 48, no. 2, pp. 155-161, 2012.

[41] Q. Li and G. M. Xu, "Scale invariance in complex seismic system and its uses in gaining precursory information before large earthquakes: importance of methodology," Physica A, vol. 392, no. 4, pp. 929-940, 2013.

[42] Y. Y. Kagan, "Observational evidence for earthquakes as a nonlinear dynamic process," Physica D, vol. 77, no. 1-3, pp. 160-192, 1994.

[43] B. Enescu and K. Ito, "Some premonitory phenomena of the 1995 Hyogo-Ken Nanbu (Kobe) earthquake: seismicity, b-value and fractal dimension," Tectonophysics, vol. 338, no. 3-4, pp. 297-314, 2001.

[44] J. Feder, Fractals, Plenum Press, New York, NY, USA, 1988.

[45] Q. Li and G. M. Xu, "Research on Hurst exponent of earthquake frequency in Jiangsu and its adjacent area," Northwestern Seismological Journal, vol. 24, pp. 247-250, 2002 (Chinese).

[46] R. Bove, V. Pelino, and L. de Leonibus, "Complexity in rainfall phenomena," Communications in Nonlinear Science and Numerical Simulation, vol. 11, no. 6, pp. 678-684, 2006.

[47] J. Li and Y. Chen, "Rescaled range (R/S) analysis on seismic activity parameters," Acta Seismologica Sinica, vol. 14, no. 2, pp. 148-155, 2001.

[48] Y. Q. Li and P. L. Wang, "Analysis on earthquake frequency Hurst exponent in active block of Qinghai-Xizhang Plateau," Plateau Earthquake Research, vol. 18, pp. 36-40, 2006 (Chinese).

[49] B. Q. Wang, H. M. Huang, H. s. Fan, C. Z. Wang, and P. Y. Chen, "Nonlinear R/S method and its applicaion in earthquake prediction," Acta Seismologica Sinica, vol. 17, pp. 528-532, 1995.

[50] A. Arneodo, G. Grasseau, and M. Holschneider, "Wavelet transform of multifractals," Physical Review Letters, vol. 61, no. 20, pp. 2281-2284, 1988.

[51] F. Liu and J. Z. Cheng, "Local fractal scale wavelet analysis," Journal of Xian Jiaotong University, vol. 33, pp. 14-34, 1999 (Chinese).

[52] F. S. Yang, Application of Wavelet Transform on Engineering Analysis, Science Press, Beijing, China, 2003, (Chinese). 

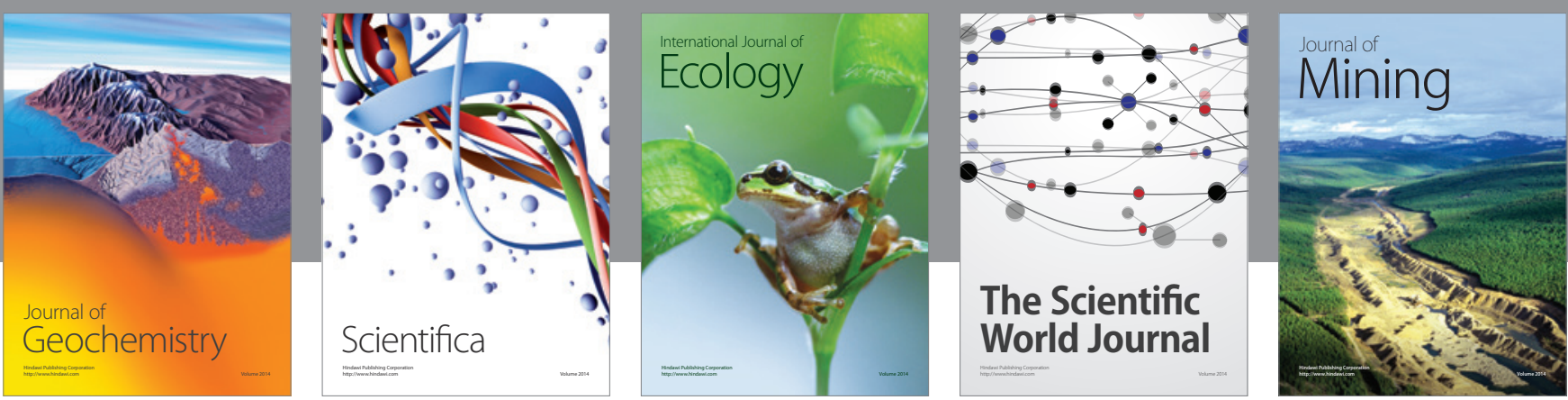

The Scientific World Journal
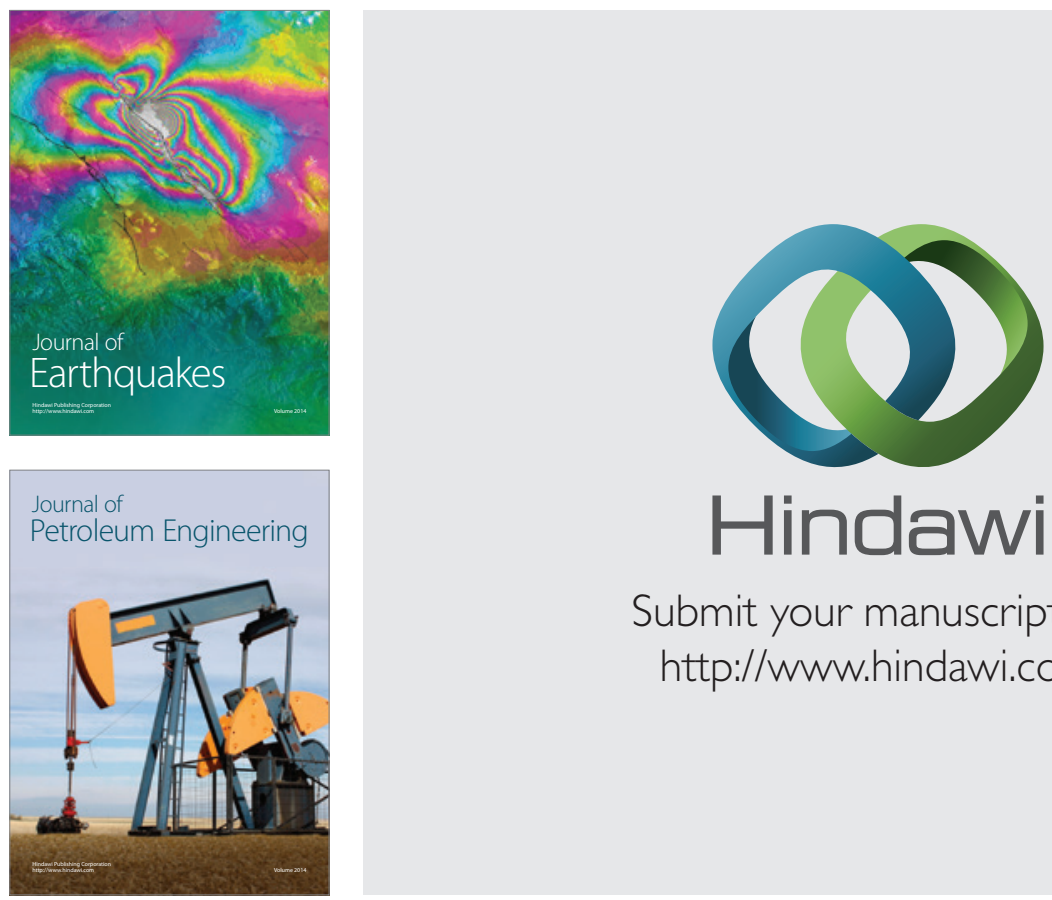

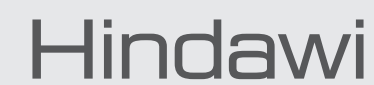

Submit your manuscripts at

http://www.hindawi.com
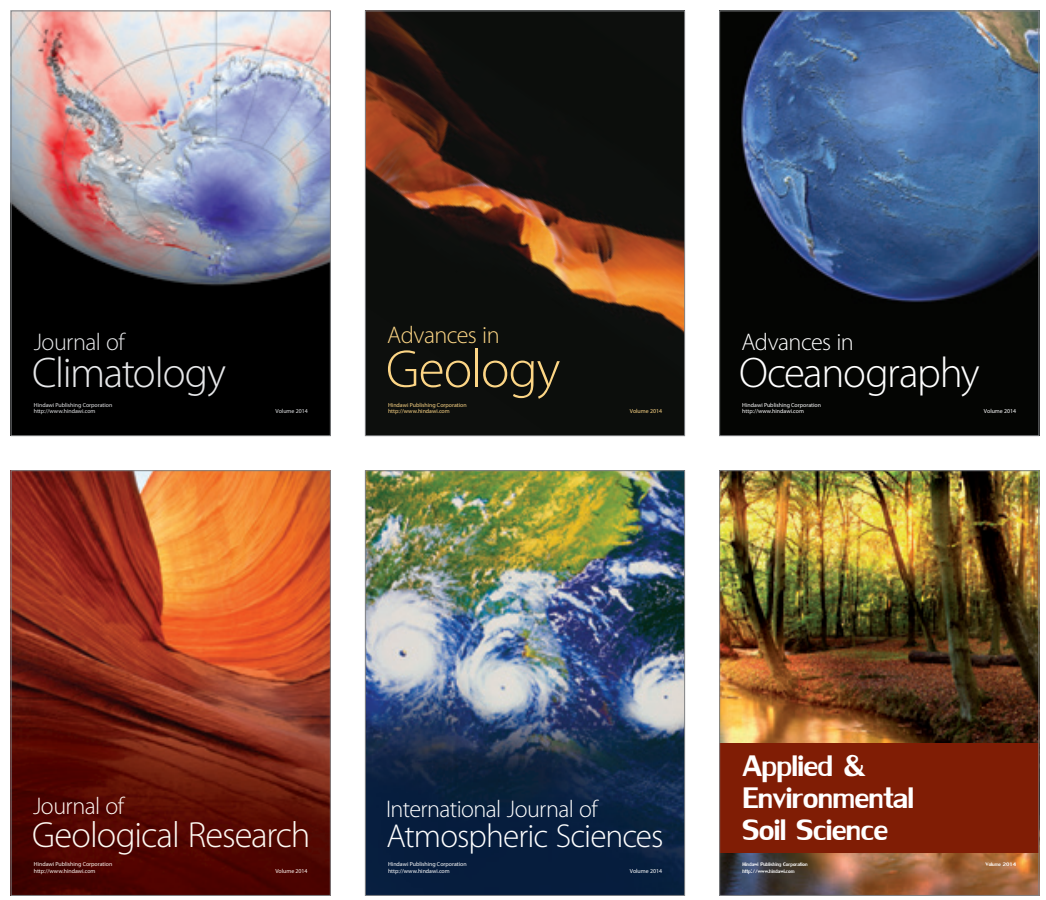
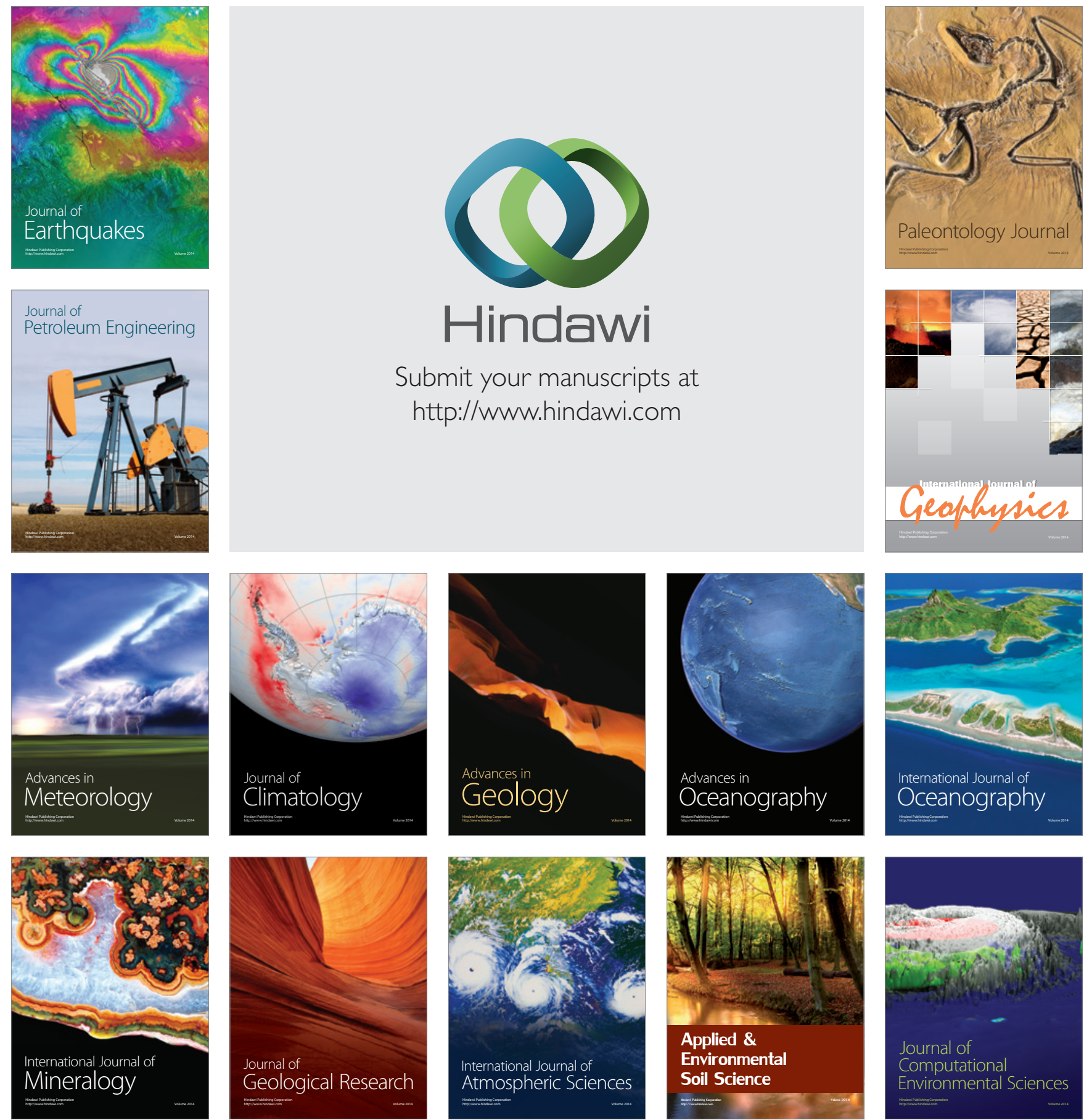